\title{
Time-varying Feedback Stabilization of the Attitude of a Rigid Spacecraft with two controls *
}

\author{
P. Morin C. Samson J.-B. Pomet Z.-P. Jiang $\dagger$ \\ INRIA, 2004 route des Lucioles, 06902 Sophia Antipolis cedex, FRANCE
}

February 16, 1995

\begin{abstract}
It is known that rigid body models with two controls cannot be locally asymptotically stabilized by continuous feedbacks which are functions of the state only. This impossibility does no longer hold when the feedback is also a function of time, or when it is discontinuous. A locally stabilizing smooth time-varying feedback is here explicitly derived by using Center Manifold Theory combined with averaging and Lyapunov techniques.
\end{abstract}

Keywords: Time-varying control, smooth feedback, attitude stabilization.

\section{Introduction}

The attitude control of a rigid spacecraft operating in degraded mode, i.e. with only one or two controls, has already been much studied in the literature. This type of system has for example been used to illustrate several aspects of nonlinear controllability. Let us mention the results of Bonnard [3] and Crouch [8] proving that the system is globally controllable except for some exceptional locations of the actuators, and the work by Kerai [11] which establishes small time local controllability (STLC) in the same situations with two controls.

The present paper focuses on the attitude stabilization problem. Some early results may be found in [8] where controllability results are used to transform open-loop control strategies into a feedback. The feedback so obtained is discontinuous and a simplified alternative method is proposed in [12]. We are concerned here with continuous feedback. The related but simpler problem consisting of stabilizing the angular velocity of the spacecraft with one or two actuators has been investigated by several authors, see Aeyels [1], Sontag and Sussmann [22], and more recently Byrnes and Isidori [2]. In the past, it seems that continuous stabilization of the attitude has been implicitly ruled out because this system belongs to the class of systems, singled out by Brockett [4], which are controllable but cannot be

\footnotetext{
*This work was supported by DRET under contract $n^{\circ}$ 931315/A000/DRET/DS/SR2.

$\dagger$ Presently at: Depart ment of Systems Engineering, Australian National University, ACT 0200, AUSTRALIA
} 
stabilized via continuous state feedback. On the other hand, an article by Samson [19] has recently triggered the discovery that many systems of interest which cannot be stabilized by continuous state-feedback can in fact be stabilized by smooth "time-varying" feedback. Research on time-varying control has then expanded quickly. In particular Coron (see e.g [6], [7]) established that, "most" STLC systems are stabilizable by continuous time-varying feedback. This result, combined with the aforementioned result by Kerai on STLC [11], yields the existence of a stabilizing continuous time-varying feedback for a rigid spacecraft with two pairs of gas jets (i.e. two controls), except in the cases known to be uncontrollable. Besides the existence issue, methods for the design of time-varying feedbacks have also been developed for driftless systems which do not satisfy Brockett's necessary condition (see e.g [17], [18]). However their extensions to systems with drift, such as the one considered here, is not straightforward. In [13], Samson and Morin present a first attempt to stabilize the spacecraft with time-varying controls. Although no stability proof is provided there, the given control laws display stabilization properties on simulations.

In the present paper, an explicit smooth time-varying feedback with proven stabilizing properties is derived ${ }^{1}$. The techniques used for the construction of this control combine center manifold theory with time-averaging and Lyapunov techniques. The idea of applying center manifold theory to time-varying stabilization has previously been used in [21], and subsequently in [23], for the stabilization of some driftless systems. A preliminary description of the present results was presented at the $33^{\text {rd }}$ IEEE CDC [14].

The paper is organized as follows. Section 2 recalls the equations of a rigid body and precisely states the control objective. A simplified control problem is treated in Section 3 and our main result is given in Section 4. Throughout the paper, we use the following notations:

- $|X|$ or $\left|x_{1}, \ldots, x_{n}\right|$ denotes the Euclidean norm of a vector $X$ or $\left(x_{1}, \ldots, x_{n}\right)$.

- $O\left(|X|^{q}\right)$ is the usual notation (see for instance [16] for more details) for any expression whose norm is bounded by $K|X|^{q}$ for a certain $K$ in a neighborhood of $X=0$. For time-varying functions, we require that $K$ be independant of time $t$. For instance, $f(X, t)=O\left(|X|^{q}\right)$ with $f$ a function from $\mathbb{R}^{n} \times \mathbb{R}$ to $\mathbb{R}^{m}$ for some $n$ and $m$ means

$$
\exists \delta>0, \exists K>0, \forall t, \quad|X|<\delta \Rightarrow|f(X, t)| \leq K|X|^{q},
$$

and $l\left(O\left(\left|x_{1}, x_{2}\right|^{2}\right), 0, x_{1}, x_{2}, t\right)=O\left(\left|x_{1}, x_{2}\right|^{3}\right)$, for $l$ a function from $\mathbb{R}^{4} \times \mathbb{R}$ to any $\mathbb{R}^{m}$ and $x_{1}$ and $x_{2}$ scalar means that $l\left(\eta\left(x_{1}, x_{2}, t\right), 0, x_{1}, x_{2}, t\right)=O\left(\left|x_{1}, x_{2}\right|^{3}\right)$ for all function $\eta$ such that $\eta\left(x_{1}, x_{2}, t\right)=O\left(\left|x_{1}, x_{2}\right|^{2}\right)$.

\section{Problem Statement}

Let us denote:

- $\omega$ : the angular velocity vector of the frame $F_{0}$ attached to the spacecraft (with axes corresponding to the principal inertia axes of the body) with respect to the frame $F_{1}$ whose attitude is the desired one for $F_{0}$. This vector is expressed in the basis of $F_{0}$.

\footnotetext{
${ }^{1}$ While preparing this manuscript, we have been informed of an independent work by R. Montgomery, S.S. Sastry, and G.C. Walsh in the same direction. No preprint has yet been communicated to us.
} 
- $J=\operatorname{diag}\left(j_{1}, j_{2}, j_{3}\right)$ : the inertia matrix of the spacecraft.

- $S(\omega)=\left(\begin{array}{ccc}0 & \omega_{3} & -\omega_{2} \\ -\omega_{3} & 0 & \omega_{1} \\ \omega_{2} & -\omega_{1} & 0\end{array}\right)$ : the matrix representation of the cross product.

If $R$ is the rotation matrix representing the attitude of $F_{1}$ with respect to $F_{0}$ (and whose columns vectors are the basis vectors of $F_{1}$ expressed in $F_{0}$ ), we get the well known equations:

$$
\left\{\begin{array}{l}
\dot{R}=S(\omega) R \\
J \dot{\omega}=S(\omega) J \omega+\left(\tau_{1}, \tau_{2}, 0\right)^{T}
\end{array}\right.
$$

where the $\tau_{i}$ are the torques applied to the rigid body.

This is a control system with two inputs $\tau_{1}$ and $\tau_{2}$ and state space $S O(3) \times \mathbb{R}^{3}$. Our objective is to find a control $\left(\tau_{1}(t, R, \omega), \tau_{2}(t, R, \omega)\right)$ periodic with respect to time, which locally asymptotically stabilizes the point $\left(I_{3}, 0\right)$ of $S O(3) \times \mathbb{R}^{3}$, with $I_{3}$ denoting the identity matrix. In order to control the body rotations, a preliminary step traditionally consists in defining a minimal set of local coordinates for the parametrization of $S O(3)$ around the identity. A common choice is the Euler angles. We make a different choice that has the advantage of yielding polynomial equations. Let $S^{3}$ be the three-dimensional sphere, identified with the multiplicative group of unitary quaternions $\lambda=\left(\lambda_{0}, \lambda_{1}, \lambda_{2}, \lambda_{3}\right)$ with $\lambda_{0}^{2}+\lambda_{1}^{2}+\lambda_{2}^{2}+\lambda_{3}^{2}=1$ and with $\lambda_{0}$ the real part of the quaternion. It is established in [20] that (1) lifts to the following control system on $S^{3} \times \mathbb{R}^{3}$ :

$$
\left\{\begin{array}{l}
\dot{\lambda}=\frac{1}{2}\left(\begin{array}{cc}
0 & -\omega^{T} \\
\omega & S(\omega)
\end{array}\right) \lambda \\
J \dot{\omega}=S(\omega) J \omega+\left(\tau_{1}, \tau_{2}, 0\right)^{T}
\end{array}\right.
$$

We choose, on the hemisphere $\lambda_{0}>0$, the following coordinates, sometimes called Rodrigues parameters:

$$
X=\left(x_{1}, x_{2}, x_{3}\right)=\left(\frac{\lambda_{1}}{\lambda_{0}}, \frac{\lambda_{2}}{\lambda_{0}}, \frac{\lambda_{3}}{\lambda_{0}}\right)
$$

The mapping $\lambda \mapsto X$ is a diffeomorphism from this hemisphere to $\mathbb{R}^{3}$, and $X$ is a minimal parameterization of the rotations of angle different from $\pi$. It is simple to write the system (2) -and hence the original system-in the coordinates $(X, \omega)$. One obtains:

$$
\left\{\begin{array}{l}
\dot{X}=\frac{1}{2}\left(\omega+S(\omega) X+\left(\omega^{T} X\right) X\right) \\
\dot{\omega}_{1}=c_{1} \omega_{2} \omega_{3}+u_{1} \\
\dot{\omega}_{2}=c_{2} \omega_{1} \omega_{3}+u_{2} \\
\dot{\omega}_{3}=c_{3} \omega_{1} \omega_{2}
\end{array}\right.
$$

with $c_{1}=\frac{j_{2}-j_{3}}{j_{1}}, c_{2}=\frac{j_{3}-j_{1}}{j_{2}}, c_{3}=\frac{j_{1}-j_{2}}{j_{3}}, u_{1}=\frac{\tau_{1}}{j_{1}}$, and $u_{2}=\frac{\tau_{2}}{j_{2}}$.

It is of course assumed that $c_{3} \neq 0$, since otherwise the system is neither controllable nor stabilizable. We may also assume that $c_{3}>0$, due to the fact that the change of variables 
$\left(x_{1}, x_{2}, x_{3}, \omega_{1}, \omega_{2}, \omega_{3}, u_{1}, u_{2}\right) \longmapsto\left(x_{2}, x_{1},-x_{3}, \omega_{2}, \omega_{1},-\omega_{3}, u_{2}, u_{1}\right)$ leaves the equation (3) unchanged, except for the parameters $\left(c_{1}, c_{2}, c_{3}\right)$ which are changed into $\left(-c_{2},-c_{1},-c_{3}\right)$.

The problem addressed in this paper consists of finding a smooth feedback control law which asymptotically stabilizes the origin $(X=0, \omega=0)$ of $(3)$. The problem is first simplified by considering the following reduced order system obtained by taking $v_{1} \triangleq \omega_{1}$ and $v_{2} \triangleq \omega_{2}$ as control variables :

$$
\left(\begin{array}{l}
\dot{x}_{1} \\
\dot{x}_{2} \\
\dot{x}_{3}
\end{array}\right)=\frac{1}{2}\left[\left(\begin{array}{l}
v_{1} \\
v_{2} \\
\omega_{3}
\end{array}\right)+\left(\begin{array}{ccc}
0 & \omega_{3} & -v_{2} \\
-\omega_{3} & 0 & v_{1} \\
v_{2} & -v_{1} & 0
\end{array}\right)\left(\begin{array}{l}
x_{1} \\
x_{2} \\
x_{3}
\end{array}\right)+\left(v_{1} x_{1}+v_{2} x_{2}+\omega_{3} x_{3}\right)\left(\begin{array}{l}
x_{1} \\
x_{2} \\
x_{3}
\end{array}\right)\right]
$$

\section{Stabilization of the reduced system with velocity controls}

A possible control law and the corresponding stabilization result are stated next:

Theorem 1 The smooth time-varying control:

$$
\left\{\begin{array}{l}
v_{1}\left(X, \omega_{3}, t\right)=2 g_{1} \dot{h}_{1}+h_{1} \frac{\partial g_{1}}{\partial x_{3}} \omega_{3}-2 k_{1}\left(x_{1}-g_{1} h_{1}\right) \\
v_{2}\left(X, \omega_{3}, t\right)=2 g_{2} \dot{h}_{2}-x_{3} v_{1}+x_{1} \omega_{3}+h_{2} \frac{\partial g_{2}}{\partial x_{3}} \omega_{3}-2 k_{2}\left(x_{2}-g_{2} h_{2}\right)
\end{array}\right.
$$

with

$$
\left\{\begin{array}{l}
g_{1}\left(x_{3}, \omega_{3}\right)=\alpha x_{3}+\beta \omega_{3}, \quad g_{2}\left(x_{3}, \omega_{3}\right)=x_{3}^{2}+\omega_{3}^{2} \\
h_{1}(t)=a_{1} \sin t, \quad h_{2}(t)=a_{2} \sin t+a_{3} \cos t
\end{array}\right.
$$

and parameters $k_{1}, k_{2}, a_{1}, a_{2}, a_{3}, \alpha$ and $\beta$ such that:

$$
k_{1}>0, \quad k_{2}>0, \quad a_{1}>0, \quad a_{2}<0, \quad a_{3}>0, \quad \alpha=-\frac{a_{3}^{2}}{8 a_{1} a_{2}}, \quad \beta=\frac{a_{3}}{4 a_{1}}
$$

locally asymptotically stabilizes the origin of (4).

The proof developed in the sequel of the section consists in a step by step construction of the control law (5)-(7).

From the first two equations of system (4), it is clearly possible to create for the closed loop system, via an adequate choice of $v_{1}$ and $v_{2}$, a two-dimensional attractive center manifold. We choose a center manifold which is time varying (in order to circumvent Brockett's necessary condition) and arbitrarily assign an approximation given by $x_{1}=$ $g_{1}\left(x_{3}, \omega_{3}\right) h_{1}(t)$ and $x_{2}=g_{2}\left(x_{3}, \omega_{3}\right) h_{2}(t)$ where $h_{1}$ and $h_{2}$ are periodic functions of time. In order to simplify the exposition, we make the following change of variables:

$$
\left\{\begin{array}{l}
z_{1}=x_{1}-g_{1}\left(x_{3}, \omega_{3}\right) h_{1}(t) \\
z_{2}=x_{2}-g_{2}\left(x_{3}, \omega_{3}\right) h_{2}(t)
\end{array}\right.
$$

The control (5) can then be rewritten in the new coordinates $\left(z_{1}, z_{2}, x_{3}, \omega_{3}\right)$ as:

$$
\left\{\begin{array}{lll}
v_{1}\left(z_{1}, x_{3}, \omega_{3}, t\right) & =2 g_{1} \dot{h}_{1}+h_{1} \frac{\partial g_{1}}{\partial x_{3}} \omega_{3}-2 k_{1} z_{1}, & k_{1}>0 \\
v_{2}\left(z_{1}, z_{2}, x_{3}, \omega_{3}, t\right) & =2 g_{2} \dot{h}_{2}-x_{3} v_{1}+\left(z_{1}+g_{1} h_{1}\right) \omega_{3}+h_{2} \frac{\partial g_{2}}{\partial x_{3}} \omega_{3}-2 k_{2} z_{2}, & k_{2}>0
\end{array}\right.
$$


Lemma 1 With the controls $v_{1}$ and $v_{2}$ given by (9), where $g_{1}$ and $g_{2}$ are homogeneous polynomials in $x_{3}$ and $\omega_{3}$ of degree 1 and 2 respectively, and $h_{1}$ and $h_{2}$ smooth T-periodic functions of time, the closed-loop system may be written, in coordinates $\left(z_{1}, z_{2}, x_{3}, \omega_{3}\right)$, as:

$$
\left\{\begin{array}{c}
\dot{z}_{1}=-k_{1} z_{1}+l_{1}\left(z_{1}, z_{2}, x_{3}, \omega_{3}, t\right) \\
\dot{z}_{2}=-k_{2} z_{2}+l_{2}\left(z_{1}, z_{2}, x_{3}, \omega_{3}, t\right) \\
\left(\begin{array}{c}
\dot{x}_{3} \\
\dot{\omega}_{3}
\end{array}\right)=\left(\begin{array}{cc}
0 & \frac{1}{2} \\
0 & 0
\end{array}\right)\left(\begin{array}{l}
x_{3} \\
\omega_{3}
\end{array}\right)+f\left(z_{1}, z_{2}, x_{3}, \omega_{3}, t\right)
\end{array}\right.
$$

where $f=\left(f_{1}, f_{2}\right)^{T}$ and $l=\left(l_{1}, l_{2}\right)^{T}$ are smooth functions, T-periodic with respect to time, which vanish at $(0,0,0,0, t)$ and whose first order derivatives also vanish at these points, and are such that:

$$
\left\{\begin{aligned}
& f_{1}\left(z_{1}, z_{2}, x_{3}, \omega_{3}, t\right)=-\frac{1}{2}\left(z_{2}+h_{2}(t) g_{2}\right) v_{1}+\frac{1}{2}\left(z_{1}+h_{1}(t) g_{1}\right) v_{2} \\
&+\frac{1}{2}\left[\left(z_{1}+h_{1}(t) g_{1}\right) v_{1}+\left(z_{2}+h_{2}(t) g_{2}\right) v_{2}+x_{3} \omega_{3}\right] x_{3} \\
& f_{2}\left(z_{1}, z_{2}, x_{3}, \omega_{3}, t\right)=c_{3} v_{1} v_{2} \\
& l_{1}\left(0,0, x_{3}, \omega_{3}, t\right)=O\left(\left|x_{3}, \omega_{3}\right|^{3}\right) \\
& l_{2}\left(O\left(\left|x_{3}, \omega_{3}\right|^{3}\right), 0, x_{3}, \omega_{3}, t\right)=O\left(\left|x_{3}, \omega_{3}\right|^{4}\right)
\end{aligned}\right.
$$

The proof of this lemma involves a tedious but straightforward verification which is not reproduced here due to the lack of space. The reader is thus referred to [15] for the details.

The closed-loop system (10) is now in the right form for the application of center manifold results. We will specifically use the following lemma, which is not completely standard in that it deals with time-varying periodic vector fields and also involves various orders of approximation of the center manifold.

Lemma 2 Consider the system

$$
\left\{\begin{array}{l}
\dot{z}=B z+l(z, x, t) \\
\dot{x}=A x+f(z, x, t)
\end{array}\right.
$$

with $z \in \mathbb{R}^{n}, x \in \mathbb{R}^{m}, B$ an upper triangular stable matrix $\left(b_{i j}=0\right.$ if $i>j, b_{i i}<0$ for $i=1, \ldots, n), A$ a matrix with eigenvalues having zero real parts, $l$ and $f$ T-periodic functions of class $C^{2}$ vanishing at $(0,0, t)$ and whose first order derivatives also vanish at this point.

Assume that there exists an ordered set of integers $q_{i}$ :

$$
2 \leq q_{1} \leq q_{2} \leq \ldots \leq q_{n}
$$

such that:

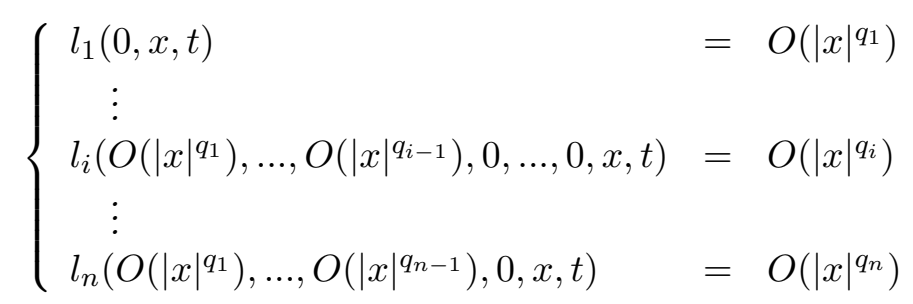

Assume further that the origin of the time-varying system:

$$
\dot{x}=A x+f\left(\pi_{1}(x, t), \ldots, \pi_{n}(x, t), x, t\right)
$$


is locally asymptotically stable when $\pi_{i}(x, t)=O\left(|x|^{q_{i}}\right),(i=1, \ldots n)$. Then, the origin of (12) is locally asymptotically stable.

(Proof in the Appendix)

In order to prove Theorem 1, one only has to show that this lemma applies, with $q_{1}=3$ and $q_{2}=4$, to the system (10) of lemma 1 for which:

$$
n=2, A=\left(\begin{array}{cc}
0 & \frac{1}{2} \\
0 & 0
\end{array}\right), B=\left(\begin{array}{cc}
-k_{1} & 0 \\
0 & -k_{2}
\end{array}\right) .
$$

To this purpose, it is sufficient to show that the origin of the following system:

$$
\left(\begin{array}{c}
\dot{x}_{3} \\
\dot{\omega}_{3}
\end{array}\right)=\left(\begin{array}{cc}
0 & \frac{1}{2} \\
0 & 0
\end{array}\right)\left(\begin{array}{l}
x_{3} \\
\omega_{3}
\end{array}\right)+f\left(\pi_{1}\left(x_{3}, \omega_{3}, t\right), \pi_{2}\left(x_{3}, \omega_{3}, t\right), x_{3}, \omega_{3}, t\right)
$$

is locally asymptotically stable for any $\pi_{1}\left(x_{3}, \omega_{3}, t\right)=O\left(\left|x_{3}, \omega_{3}\right|^{3}\right)$ and $\pi_{2}\left(x_{3}, \omega_{3}, t\right)=$ $O\left(\left|x_{3}, \omega_{3}\right|^{4}\right)$. Let us hereafter concentrate on system (16). Using the fact that, from(9),

$$
\left\{\begin{array}{l}
v_{1}\left(O\left(\left|x_{3}, \omega_{3}\right|^{3}\right), x_{3}, \omega_{3}, t\right)-\tilde{v}_{1}\left(x_{3}, \omega_{3}, t\right)=O\left(\left|x_{3}, \omega_{3}\right|^{3}\right) \\
v_{2}\left(O\left(\left|x_{3}, \omega_{3}\right|^{3}\right), O\left(\left|x_{3}, \omega_{3}\right|^{4}\right), x_{3}, \omega_{3}, t\right)-\tilde{v}_{2}\left(x_{3}, \omega_{3}, t\right)=O\left(\left|x_{3}, \omega_{3}\right|^{4}\right)
\end{array}\right.
$$

with

$$
\left\{\begin{array}{l}
\tilde{v}_{1}\left(x_{3}, \omega_{3}, t\right)=2 g_{1} \dot{h}_{1}+h_{1} \frac{\partial g_{1}}{\partial x_{3}} \omega_{3} \\
\tilde{v}_{2}\left(x_{3}, \omega_{3}, t\right)=2 g_{2} \dot{h}_{2}-x_{3} \tilde{v}_{1}+g_{1} h_{1} \omega_{3}+h_{2} \frac{\partial g_{2}}{\partial x_{3}} \omega_{3}
\end{array}\right.
$$

we may, from (11), rewrite (16) as :

$$
\left\{\begin{array}{l}
\dot{x}_{3}=\frac{1}{2}\left(\omega_{3}-g_{2} h_{2} \tilde{v}_{1}+g_{1} h_{1} \tilde{v}_{2}+x_{3}^{2} \omega_{3}+x_{3} g_{1} h_{1} \tilde{v}_{1}\right)+O\left(\left|x_{3}, \omega_{3}\right|^{5}\right) \\
\dot{\omega}_{3}=c_{3} \tilde{v}_{1} \tilde{v}_{2}+O\left(\left|x_{3}, \omega_{3}\right|^{5}\right) .
\end{array}\right.
$$

In order to facilitate the study of this time-varying system, it is possible to introduce a time-varying change of coordinates, the effect of which is to render all terms, up to the fourth order, time-invariant. This is precisely stated in the following lemma which is reminiscent of classical results, reported for example in [9], and similar results used in [23].

Lemma 3 (Averaging lemma) Let $A$ be $a n \times n$ strictly upper triangular matrix $(j \leq$ $\left.i \Rightarrow a_{i j}=0\right), D(t) a n \times p$ matrix whose components $d_{i j}(t)$ are $T$-periodic functions of class $C^{r}(r \geq 0)$, and $\sigma(x)$ a vector-valued function whose components $\sigma_{i}\left(x_{1}, \ldots, x_{n}\right)$ are monomials of degree $k \geq 2$.

Then there exists a neighborhood $\Omega$ of 0 in $\mathbb{R}^{n}$ and a $C^{r+1}$ local change of coordinates $(x, t) \mapsto(y, t)$ defined on $\Omega \times \mathbb{R}$ which maps $(0, t)$ into itself and transforms a system:

$$
\dot{x}=A x+D(t) \sigma(x)+O\left(|x|^{2 k-1}\right)
$$

into a system:

$$
\dot{y}=A y+\bar{D} \sigma(y)+O\left(|y|^{2 k-1}\right)
$$

where $\bar{D}$ is the time average of $D(t)$, i.e.. $\bar{D}=\frac{1}{T} \int_{0}^{T} D(t) d t$. 
(Proof in the Appendix)

This lemma is here applied, with $k=3$, to the system (19) for which $n=2$ and $x=\left(x_{3}, \omega_{3}\right)$. When writing the system (19) in the form (20), the right hand side of (19) contains linear terms associated with the matrix $A$, terms of order at least equal to five regrouped in the vector $O\left(|x|^{2 k-1}\right)$, and terms of order 3 with time-periodic coefficients forming the term $D(t) \sigma(x)$. One may take $\sigma\left(x_{3}, \omega_{3}\right)=\left(x_{3}^{3}, x_{3}^{2} \omega_{3}, x_{3} \omega_{3}^{2}, \omega_{3}^{3}\right)^{T}$ and determine the time-varying matrix $D(t)$ accordingly. In view of this lemma, it is equivalent to study the stability of the system (19) and the stability of the corresponding "averaged" system which, after some calculations using the particular choice (6) for the $2 \pi$-periodic $(T=2 \pi)$ functions $g_{i}$ and $h_{i}(i=1,2)$, can be written (see [15] for the details):

$$
\left\{\begin{aligned}
\dot{x}_{3}= & \frac{1}{2} \omega_{3}-2 \alpha L x_{3}^{3}+\omega_{3} O\left(\left|x_{3}, \omega_{3}\right|^{2}\right)+O\left(\left|x_{3}, \omega_{3}\right|^{5}\right) \\
\dot{\omega}_{3}=c_{3}\left[\omega_{3}^{3}(-2 \alpha L-4 \beta M+N \alpha \beta)\right. & \\
& +\omega_{3}^{2} x_{3}\left(-6 \alpha M+4 \beta L-4 N \beta^{2}\right) \\
& +\omega_{3} x_{3}^{2}(2 \alpha L-4 \beta M-8 N \alpha \beta) \\
& \left.+x_{3}^{3}\left(-4 \alpha M-4 N \alpha^{2}\right)\right]+O\left(\left|x_{3}, \omega_{3}\right|^{5}\right)
\end{aligned}\right.
$$

with

$$
L=\frac{a_{1} a_{3}}{2}, M=-\frac{a_{1} a_{2}}{2}, N=\frac{a_{1}^{2}}{2}
$$

Note that for any given $\operatorname{triplet}(L, M, N)$ with $N>0$, there exists a corresponding triplet $\left(a_{1}, a_{2}, a_{3}\right)$ of real numbers such that (23) is verified. Consequently, the numbers $L, M$ and $N$ may in turn be interpreted as independent design parameters.

From there, there only remains to show that, for some values of $\alpha, \beta, L, M$ and $N$, the origin of the system (22) is asymptotically stable.

Lemma 4 Consider the function $V\left(x_{3}, \omega_{3}\right)=2 N c_{3}\left(\alpha^{2}+2 \beta^{2}\right) x_{3}^{4}+\frac{1}{2} \omega_{3}^{2}$. If

$$
L>0, \quad M>0, \quad N>0, \quad \beta=\frac{L}{4 N} \quad \text { and } \quad \alpha=\frac{2 N \beta^{2}}{M},
$$

then, there exists $K>0$ such that, in a neighborhood of $\left(x_{3}=0, \omega_{3}=0\right)$, we have along any solution of (22):

$$
\frac{d V}{d t} \leq-K V^{2}
$$

The proof of this lemma is a straightforward verification of the inequality (25). It is not reproduced here due to the lack of space (see [15] for the details).

From this lemma, one deduces, by application of "Lyapunov second method", that the origin of (22) is locally asymptotically stable when the conditions (24) are met. Since it is so when choosing the control parameters according to (7), this proves Theorem 1.

\section{Stabilization with torque controls}

We now return to the problem of stabilizing the origin of the initial system (3) with $u_{1}$ and $u_{2}$, instead of $\omega_{1}$ and $\omega_{2}$, as control inputs. Note that since $u_{1}+c_{1} \omega_{2} \omega_{3}=\dot{\omega}_{1}$, and 
$u_{2}+c_{2} \omega_{1} \omega_{3}=\dot{\omega}_{2}$, the problem is basically equivalent to the one associated with the use of $\dot{\omega}_{1}$ and $\dot{\omega}_{2}$ as control inputs. This corresponds to the classical situation where integrators are added at the input level. The result, which is deduced from Theorem 1, is the following.

Theorem 2 The smooth time-varying control law:



with $v_{1}$ and $v_{2}$ given by (5), (6) and (7) and, $s_{1}$ and $s_{2}$ their time derivatives along the trajectories of (3):

$$
s_{i}=\frac{1}{2} \frac{\partial v_{i}}{\partial X}\left(\omega+S(\omega) X+\left(\omega^{T} X\right) X\right)+\frac{\partial v_{i}}{\partial \omega_{3}} c_{3} \omega_{1} \omega_{2}+\frac{\partial v_{i}}{\partial t} \quad i=1,2 .
$$

locally asymptotically stabilizes the origin of (3).

The techniques used here are standard in the time-invariant case. This theorem can be proved by using the following lemma, which may be viewed as a particular case of the small-gain theorem for nonlinear systems (see [24, Fact 4.1, Lemma 4.1]).

Lemma 5 Consider the T-periodic system

$$
\left\{\begin{array}{l}
\dot{x}=f(x, y, t), \quad x \in \mathbb{R}^{n} \\
\dot{y}=g(y, t), \quad y \in \mathbb{R}^{m}
\end{array}\right.
$$

where $f$ and $g$ are functions of class $C^{1}$. Assume that the origins of the two independent systems $\dot{x}=f(x, 0, t)$ and $\dot{y}=g(y, t)$ are locally asymptotically stable. Then the origin $(x=0, y=0)$ of the cascaded system (27) is locally asymptotically stable.

Setting $\tilde{\omega}=\omega-\eta$ with $\eta=\left(v_{1}, v_{2}, 0\right)$, the system (3) subjected to the controls (26) can be rewritten in the new coordinates $(X, \tilde{\omega})$ :

$$
\left\{\begin{array}{l}
\dot{X}=\frac{1}{2}\left[\tilde{\omega}+\eta+S(\tilde{\omega}+\eta) X+\left((\tilde{\omega}+\eta)^{T} X\right) X\right] \\
\dot{\tilde{\omega}}_{3}=c_{3}\left(\tilde{\omega}_{1}+\eta_{1}\right)\left(\tilde{\omega}_{2}+\eta_{2}\right) \\
\dot{\tilde{\omega}}_{1}=-k_{3} \tilde{\omega}_{1} \\
\dot{\tilde{\omega}}_{2}=-k_{4} \tilde{\omega}_{2}
\end{array}\right.
$$

By applying lemma 5 to the closed-loop system $(28)$, with $x=\left(X, \tilde{\omega}_{3}\right)$ and $y=\left(\tilde{\omega}_{1}, \tilde{\omega}_{2}\right)$, the asymptotic stability of the origin of this system stems from the asymptotic stability, previously established in Theorem 1 , of the reduced system obtained by setting $y=0$.

\section{Appendix : Proof of Lemmas 2 and 3}

\section{Proof of Lemma 2}

The proof of this lemma involves an extension of classical results of center manifold theory to the case time-periodic systems. 
Proposition 1 (Existence of a center manifold) Consider the system:

$$
\left\{\begin{array}{l}
\dot{z}=B z+l(z, x, t) \\
\dot{x}=A x+f(z, x, t)
\end{array}\right.
$$

with $z \in \mathbb{R}^{n}, x \in \mathbb{R}^{m}, B$ a matrix with eigenvalues having negative real parts, $A$ a matrix with eigenvalues having zero real parts, $l$ and $f$ T-periodic functions of class $C^{2}$ vanishing at $(0,0, t)$ and whose first partial derivatives also vanish at these points. Then, there exists $\delta>0$ and $\pi(x, t): \mathbb{R}^{m} \times \mathbb{R} \longrightarrow \mathbb{R}^{n}$, a T-periodic function of class $C^{2}$, such that the set $\{(\pi(x, t), x)|x|<\delta, t \in \mathbb{R}\}$ defines a local center manifold for (29), i.e.

i) $\pi$ vanishes at $(0, t)$ and its first partial derivatives also vanish at these points,

ii) solutions of (29) starting in the set $\{(\pi(x, t), x)|x|<\delta, t \in \mathbb{R}\}$ remain in this set as long as $|x(t)|<\delta$, i.e.

$$
\frac{\partial \pi}{\partial t}+\frac{\partial \pi}{\partial x}(x, t)(A x+f(\pi(x, t), x, t))=B \pi(x, t)+l(\pi(x, t), x, t) \quad \text { for }|x|<\delta .
$$

This result is a particular case of [10, Th 1 and section 5].

Proposition 2 (Reduction principle) Let $\pi$ be a function satisfying the conditions i) and ii) of Proposition 1. If the origin of the corresponding "reduced system" :

$$
\dot{x}=A x+f(\pi(x, t), x, t)
$$

is locally asymptotically stable, then so is the origin of (29). Moreover, in this case, if $\left(z_{0}, x_{0}\right)$ is small enough, and $(z(t), x(t))$ is the solution of (29) with initial values $\left(z_{0}, x_{0}\right)$ at $t=t_{0}$, then there exists a solution $u(t)$ of (31) such that:

$$
\left\{\begin{array}{l}
x(t)-u(t)=O\left(e^{-\gamma t}\right) \\
z(t)-\pi(u(t), t)=O\left(e^{-\gamma t}\right)
\end{array}\right.
$$

where $\gamma$ depends only on the eigenvalue of $B$ with largest real part.

Proposition 3 (Approximation) Let $\pi$ be a function satisfying the conditions i) and ii) of Proposition 1, and $\Phi: \mathbb{R}^{m} \times \mathbb{R} \longrightarrow \mathbb{R}^{n}$ be a T-periodic function of class $C^{1}$ such that:

i) $\Phi$ vanishes at $(0, t)$ and its first partial derivatives also vanish at these points,

ii) $\frac{\partial \Phi}{\partial x}(x, t)[A x+f(\Phi(x, t), x, t)]-B \Phi-l(\Phi(x, t), x, t)+\frac{\partial \Phi}{\partial t}=O\left(|x|^{q}\right)$.

Then, $\pi(x, t)-\Phi(x, t)=O\left(|x|^{q}\right)$.

For the proof of these two propositions, we refer to [5]. The main reason why the proofs still hold in the time-periodic case is that the relations (2.3.5) and (2.4.10) in [5] are not modified by the introduction of the time variable when $l$ and $f$ are time-periodic. 
We now proceed with the proof of Lemma 2. From Proposition 1, there exists for system (12) a center manifold defined by $z_{1}=\pi_{1}(x, t), \ldots, z_{n}=\pi_{n}(x, t)$. If we prove that $\pi_{i}(x, t)=O\left(|x|^{q_{i}}\right)$ for $i=1, \ldots, n$, then, Lemma 2 directly follows from Proposition 2 . Let us proceed by induction.

First, it follows from (13) and (14) that $l(0, x, t)=O\left(|x|^{q_{1}}\right)$ and, by application of Proposition 3 with $\Phi=0$, we deduce that $\pi_{i}(x, t)=O\left(|x|^{q_{1}}\right)$, for $i=1, \ldots, n$. In particular, $\pi_{1}(x, t)=O\left(|x|^{q_{1}}\right)$. Let us now assume that $\pi_{1}(x, t)=O\left(|x|^{q_{1}}\right), \ldots, \pi_{k}(x, t)=O\left(|x|^{q_{k}}\right)$ and prove that $\pi_{k+1}(x, t)=O\left(|x|^{q_{k+1}}\right)$. To this purpose, we consider the following reduced-order system:

$$
\begin{array}{rccc}
\dot{z}_{k+1} & = & b_{k+1, k+1} z_{k+1}+\ldots+b_{k+1, n} z_{n}+l_{k+1}\left(\pi_{1}(x, t), \ldots, \pi_{k}(x, t), z_{k+1}, \ldots, z_{n}, x, t\right) \\
& \vdots & \ddots & \\
\dot{z}_{n} & = & b_{n, n} z_{n}+l_{n}\left(\pi_{1}(x, t), \ldots, \pi_{k}(x, t), z_{k+1}, \ldots, z_{n}, x, t\right) \\
\dot{x} & = & A x+f\left(\pi_{1}(x, t), \ldots, \pi_{k}(x, t), z_{k+1}, \ldots, z_{n}, x, t\right)
\end{array}
$$

where the $b_{i, j}$ 's are the entries of $B$ (recall that it is upper-triangular and $b_{i, i}<0$ ).

Since $(x, t) \mapsto\left(\pi_{k+1}(x, t), \ldots, \pi_{n}(x, t)\right)$ satisfies the conditions i) and ii) of Proposition 1 , and since $\Phi=0$ satisfies the conditions i) and ii) of Proposition 3 (from (13), (14) and the induction hypothesis), Proposition 3 applies with $z$ replaced by $\left(z_{k+1}, \ldots, z_{n}\right), \pi$ replaced by $\left(\pi_{k+1}, \ldots, \pi_{n}\right), \Phi=0$ and $q=q_{k+1}$. Hence, $\pi_{j}(x, t)=O\left(|x|^{q_{k+1}}\right)$, for $j=k+1, \ldots, n$, and in particular $\pi_{k+1}(x, t)=O\left(|x|^{q_{k+1}}\right)$.

\section{Proof of Lemma 3}

The proof is given for $n=2$ and $k=3$ (the spacecraft's case). The general case is a simple extension.

We assume, without loss of generality, that the components of the vector $\sigma(x)$ in (20) form a basis for the homogeneous polynomials of degree three. We take $\sigma(x)=\left(x_{1}^{3}, x_{1}^{2} x_{2}, x_{1} x_{2}^{2}, x_{2}^{3}\right)$. The change of coordinates evoked in the lemma is of the same type as the one used in [23], or originally in [9, pp 168], and is of the form $x=y+H(t) \sigma(y)$, with $H$ a $2 \times 4$ matrix of T-periodic functions which are specified further in the proof. This change of coordinates transforms (20) into:

$$
\begin{aligned}
\dot{y}= & {\left[I_{2}+H(t) \sigma^{\prime}(y)\right]^{-1}[A y+A H(t) \sigma(y)+D(t) \sigma(y+H(t) \sigma(y))} \\
& \left.-\dot{H}(t) \sigma(y)+O\left(|y|^{5}\right)\right] .
\end{aligned}
$$

(where $\sigma^{\prime}(y)$ is the Jacobian matrix of $\sigma(y)$ and $I_{2}$ is the identity matrix). Note that $\left[I_{2}+H(t) \sigma^{\prime}(y)\right]^{-1}$ is defined in a neighborhood of $y=0$ for all $t$.

Using a classical formula on matrix inverses, and the fact that $\sigma(y)=O\left(|y|^{3}\right)$, we easily obtain from (34):

$$
\begin{aligned}
\dot{y}= & {\left[I_{2}-H(t) \sigma^{\prime}(y)+O\left(|y|^{4}\right)\right][A y+A H(t) \sigma(y)+D(t) \sigma(y)-\dot{H}(t) \sigma(y)} \\
& \left.+O\left(|y|^{5}\right)\right] \\
= & A y+\bar{D} \sigma(y)+Z(t, y)+O\left(|y|^{5}\right)
\end{aligned}
$$


with

$$
\begin{aligned}
& Z(t, y)=\tilde{D}(t) \sigma(y)+A H(t) \sigma(y)-\dot{H}(t) \sigma(y)-H(t) \sigma^{\prime}(y) A y, \\
& \bar{D}=\left(\frac{1}{T} \int_{0}^{T} D(t) d t\right), \\
& \tilde{D}(t)=D(t)-\bar{D} .
\end{aligned}
$$

In order to prove the lemma, it is sufficient to find a uniformly bounded matrix $H(t)$ for which $Z(t, y) \equiv 0$. Since the entries of $\sigma^{\prime}(y) A y$ are homogeneous polynomials of degree 3 , there exists a (unique) $4 \times 4$ matrix $C$ such that $\sigma^{\prime}(y) A y=C \sigma(y)$. Since $A$ is strictly upper triangular, one easily verifies that $C$ is strictly upper triangular too. Therefore, $Z(t, y)$ is identically zero if and only if $H(t)$ is a solution of

$$
\dot{H}=\widetilde{D}(t)+A H-H C .
$$

Using the triangular structure of $A$ and $C$ and the periodicity of $\tilde{D}(t)$, one may consider a recursive construction which starts with $h_{21}(t)=\int_{0}^{t} \tilde{d}_{21}(s) d s-\frac{1}{T} \int_{0}^{T}\left(\int_{0}^{u} \tilde{d}_{21}(s) d s\right) d u$ and continues with $h_{22}(t), \ldots, h_{24}(t), h_{11}(t), \ldots, h_{14}(t)$ respectively. One easily verifies that the solution so obtained is $T$-periodic and uniformly bounded.

\section{References}

[1] D. Aeyels, Stabilization by smooth feedback of the angular velocity of a rigid body, Systems and Control Letters 5 (1985) 59-64.

[2] C.I. Byrnes, A. Isidori, On the attitude stabilization of rigid spacecraft, Automatica 27 (1991) 87-95.

[3] B. Bonnard, Controllability of mechanical control systems on Lie groups, report 82-08, L.A.G., E.N.S.I.E.G., St. Martin d'Hères, France (1982).

[4] R.W. Brockett, Asymptotic stability and feedback stabilization, in: R.W. Brockett, R.S. Millman and H.H. Sussmann Eds., Differential Geometric Control Theory (1983).

[5] J. Carr, Application of Center Manifold Theory (Springer Verlag, 1981).

[6] J.M. Coron, Global asymptotic stabilization for controllable systems without drifts, Mathematics of Control, Signal and Systems 5 (1992) 295-312.

[7] J.M. Coron, On the stabilization in finite time of locally controllable systems by means of continuous time-varying feedback laws, preprint, CMLA (1992).

[8] P.E. Crouch, Spacecraft attitude control and stabilization: applications of geometric control theory to rigid body models, IEEE Trans. Automat. Control 29 (1984) 321-331.

[9] J. Guckenheimer, P. Holmes, Nonlinear Oscillations, Dynamical systems and Bifurcations of Vector fields (Springer Verlag, 1983).

[10] A. Kelley, The stable, center stable, center, center unstable, unstable manifolds, Journal of Differential Equations 3 (1967) 546-570. 
[11] E. Kerai, Analysis of small time local controllability of the rigid body model, preprint, CMLA (1993).

[12] H. Krishnan, M. Reynahoglu, H. McClamroch, Attitude stabilization of a rigid spacecraft using gas jets actuators operating in a failure mode, Proc. 31st IEEE Conf. on Decision and Control (1992) 1612-1619.

[13] P. Morin: "Robotique spatiale: commande en orientation et en vitesses angulaires d'un satellite", Stage d'option, Ecole des Mines de Paris, 1992.

[14] P. Morin, C. Samson, J.-B. Pomet and Z.-P. Jiang, Time-varying feedback stabilization of the attitude of a rigid spacecraft with two controls, 33rd IEEE Conf. on Decision and Control (december 1994)

[15] P. Morin, C. Samson, J.-B. Pomet and Z.-P. Jiang, Time-varying feedback stabilization of the attitude of a rigid spacecraft with two controls, INRIA report, 1994.

[16] F. W. J. Olver, Asymptotics and special functions, (Academic Press, Inc., 1974).

[17] J.-B. Pomet, Explicit design of time-varying stabilizing control laws for a class of controllable systems without drift, Systems and Control Letters 18 (1992) 147-158.

[18] J.-B. Pomet, C. Samson, Exponential stabilization of nonholonomic systems in power form, IFAC Symposium on Robust Control Design (1994).

[19] C. Samson, Velocity and torque feedback control of a nonholonomic cart, Int Workshop in Adaptative and Nonlinear Control: Issues in Robotics, Grenoble, France (1990). Proc. in Advanced Robot Control 162 (Springer Verlag, 1991).

[20] C. Samson, M. Leborgne and B. Espiau, Robot Control: The Task function Approach, Oxford Engineering Science Series No. 22, (Oxford University Press, 1991).

[21] R. Sépulcre, G. Campion, V.Wertz, Some remarks on periodic feedback stabilization, 2nd IFAC NOLCOS, Bordeaux, France (1992) 418-423.

[22] E.D. Sontag and H.J. Susmann, Further comments on the stabilizability of the angular velocity of a rigid body, Systems and Control Letters 12 (1988) 213-217.

[23] A.R. Teel, R.M. Murray, G. Walsh, Nonholonomic control systems: from steering to stabilization with sinusoids, Proc. 31st IEEE Conf.on Decision and Control (1992) 1603-1609.

[24] A.R. Teel and L. Praly, Tools for semi-global stabilization by partial-state and output feedback, SIAM J. Control and Optimization, to appear. 